\title{
Metodología de la indagación aplicada a la enseñanza del diseño de intercambiadores de calor tipo Coraza-Tubo usando un OVA
}

\author{
Sergio Alejandro Carrión-Avellaneda ${ }^{a}$, Laura Angélica Ardila-Castro ${ }^{a}$ \& Camilo Andrés Ramírez-Mendoza ${ }^{b}$ \\ ${ }^{a}$ Programa de Ingeniería Mecánica, Escuela Colombiana de Ingeniería Julio Garavito, Bogotá, Colombia. sacarriona@unal.edu.co, laura.ardila.c@gmail.com \\ ${ }^{b}$ Programa de Ingeniería Industrial, Escuela Colombiana de Ingeniería Julio Garavito, Bogotá, Colombia. cami.ramirez9208@gmail.com
}

\begin{abstract}
Resumen- Los intercambiadores de calor son comúnmente usados en diversos procesos térmicos en la industria colombiana, el estudiante de ingeniería mecánica, aprende los conceptos básicos de estos dispositivos, en la asignatura de transferencia de calor. Por tal razón, los autores del presente estudio propusieron un nuevo método de enseñanza-aprendizaje, basado en tres conceptos, la metodología de la indagación, el diseño térmico de intercambiadores de calor tipo coraza-tubos y la aplicación de un objeto virtual de aprendizaje. El objetivo de este nuevo método, fue que el estudiante adquiera un rol activo en su formación y el profesor sea una guía en dicho proceso. En la primera parte del artículo, se definió la nueva metodología propuesta; luego se explicó los tres productos obtenidos, a saber, dos guías de enseñanza, una para el estudiante y otra para el profesor, y un programa de diseño de intercambiadores. En la sección de discusión, se explicó la importancia de la nueva metodología y de la herramienta computacional.
\end{abstract}

Palabras Clave - Metodología de la indagación; diseño térmico; objeto virtual de aprendizaje; intercambiador de calor.

Recibido para revisar Agosto 9 de 2017, aceptado Octubre 17 de 2017, versión final Octubre 27 de 2017

\section{Indagation methodology applied to the teaching of design of heat exchangers type shell and tubes using a VLO}

Abstract - The heat exchangers are commonly used in many thermal processes in the Colombian industry, the mechanical engineer must learn the basic concepts about these devices in the subject heat transfer. Thus, the authors of the present document suggested a new teaching-learning method based on three key concepts, indagation methodology, shell-tube heat exchangers design and the application of a virtual learning object. The aim of this new methodology was that the student acquires an active role in his formation and the teacher could be a guide on this process. In the first part of the article, it was defined the proposed new methodology, then was explain the products obtained, namely, two teaching guides, one for the student, the other for the teacher, and a program of heat exchangers design. In the discussion section, it was explained the importance of the new methodology and the computational tool.

Keywords - Indagation methodology; thermal design; virtual learning object; heat exchanger.

\section{Introducción}

Dentro de la formación profesional de un ingeniero mecánico, se encuentra el aprender a diseñar intercambiadores de calor, y aunque hay mucha información al respecto, no existe una metodología o un proceso establecido para dicho aprendizaje, es por esto, que los autores en su preocupación por facilitar la enseñanza de estos dispositivos térmicos, elaboran un proceso de enseñanza-aprendizaje, que guíe a los estudiantes y docentes en esta competencia de aprendizaje.

Este nuevo proceso, se basa en tres conceptos fundamentales, a saber, metodología de la indagación, diseño térmico y Objeto Virtual de Aprendizaje (OVA), en la que los tres conceptos se integran en una guía de tal manera que el estudiante, con el apoyo del docente, lleve a cabo un proceso de enseñanza-aprendizaje adecuado en el diseño de intercambiadores de calor.

La metodología de indagación científica, es un método de aprendizaje que conduce a la compresión y explicación, a través de la interacción directa con el mundo y la recolección directa de datos que ayudan a evaluar las posibles explicaciones de un fenómeno [1]. Esta compresión se fundamenta en preguntas que pueden tener cualquier origen, el docente, la lectura o de algún problema del mundo real. Estas preguntas son asumidas como propias del estudiante, debido a su interés en la solución del problema inicial que se le plantea [2], en este caso el diseño de un intercambiador. Con éste método el estudiante desarrolla habilidades tales como: preguntar, recoger datos, revisar evidencia con base a lo que se conoce, extraer conclusiones y discutir resultados [3], las cuales le permiten abordar un problema de cualquier índole y darle una solución pertinente.

Aunque la indagación se utiliza actualmente con mucha frecuencia, no es un concepto nuevo, ya que parte de la curiosidad del individuo, lo importante de ella, en el caso de la educación, es que parte del rol activo del estudiante en el desarrollo de sus ideas [4]; ya que es el alumno quien las madura progresivamente mientras aprende a investigar, para así construir conocimiento y comprensión [5]. A partir de esto, se llega a que el objetivo principal de la indagación como metodología pedagógica es que los alumnos aprenden a aprender, para esto el docente tiene en cuenta dos factores importantes, el aprendizaje conceptual y el entendimiento de cómo procede el estudiante para aprender, entonces, gracias a los conceptos el alumno puede dar solución al problema, mientras que, al formular preguntas e inquietudes gracias a la metodología de la indagación, el estudiante desarrolla un proceso de aprendizaje propio y adecuado para él [2].

Como citar este artículo: Carrión-Avellaneda, S.A., Ardila-Castro, L.A. and Ramírez-Mendoza, C.A., Metodología de la indagación aplicada a la enseñanza del diseño de intercambiadores de calor tipo Coraza-Tubo usando un OVA. Educación en Ingeniería, 13(25), pp. 64-71, Febrero, 2018. 
Esta metodología de indagación, específicamente en esta investigación, se orienta a que el estudiante formule preguntas respecto al diseño térmico de un intercambiador de calor, por tal razón, debe ser entendido con claridad. Un intercambiador de calor se define como una máquina térmica que aumenta la temperatura de un fluido a baja temperatura con uno a mayor temperatura, o viceversa, por medio de un intercambio de energía [6]. Debido a que esta transferencia de calor se puede dar de diferentes maneras, existen diferentes tipos de intercambiadores, todo esto ligado a los requerimientos energéticos y las características de los fluidos. De acuerdo con lo anterior se elige un tipo determinado, dentro de los más comunes están: tubo doble, tubos y coraza, placas paralelas, regenerativos y compactos [7].

El diseño térmico al cual se dirige la metodología, es el de intercambiadores de calor tipo coraza-tubos, en donde los fluidos son separados por la pared de una tubería, la trasferencia se da del fluido a la pared interna del tubo por convección, luego a lo largo del tubo por conducción y finalmente de la pared externa del tubo al otro fluido por convección [8]. Para su diseño, se tiene en cuenta los siguientes tópicos, el cálculo del coeficiente de trasferencia, que permite determinar la geometría del dispositivo térmico, a partir del calor que debe ser transferido de un fluido a otro; la temperatura media logarítmica, que es el diferencial de temperatura que permitirá el flujo de calor, de un fluido a otro; y el calor total que determina la potencia que generará el intercambiador de calor [6].

Uno de los factores más relevantes dentro del diseño, es el área de transferencia, que permite conocer detalles de la geometría del intercambiador, tales como los diámetros de tubería que deben corresponder a la escala comercial [9] y la configuración geométrica de los mismos, es decir, la ubicación dentro de la coraza [10]. Finalmente, se debe calcular la efectividad que es la capacidad que tiene el intercambiador de calor para transferir energía de un fluido a otro, que está ligada a la relación adimensional conocida como el Número de Unidades de Transferencia (NTU), que determina la capacidad del intercambiador [6]. El diseño térmico es un desarrollo sistemático en donde todas las variables se relacionan entre sí, lo que hace indispensable la compresión de los conceptos y los objetivos del diseño a realizar.

Para apoyar al proceso de enseñanza-aprendizaje del diseño de intercambiadores de calor tipo coraza-tubos, se hace uso de un Objeto Virtual de Aprendizaje (OVA), que de acuerdo con el Ministerio de Educación Nacional de Colombia es un material estructurado de forma significativa, digital dirigido al aprendizaje que es usado para el desarrollo de contenidos. Un OVA es catalogado dentro de los siguientes tipos: simuladores, cursos y/o tutoriales multimedia, animaciones, videos, documentos interactivos y colecciones de imágenes estáticas [11]. A pesar de ser una herramienta útil, cuando se implementa un OVA en la metodología de la clase, no se trata únicamente de usar un programa y seguir haciendo lo mismo en el aula, el uso de éstos avances es un incentivo para hacer uso de los aciertos de la pedagogía contemporánea y proponer nuevas técnicas de enseñanza [12]. Con esta herramienta el estudiante desarrolla habilidades tales como el auto aprendizaje y el constructivismo que le permiten llegar de manera autónoma al conocimiento que quiere adquirir [13]. Además, el trabajo colaborativo es uno de los beneficios que ofrecen éstas herramientas, ya que al estar disponible el recurso para todos los alumnos les permite compartir ideas entre ellos no sólo respecto al manejo del programa, sino de los conceptos en sí de clase, permitiéndole al estudiante que aprenda a sus propio ritmo [14].

Una de las ventajas más relevantes en el uso de un OVA es el aprendizaje autónomo, que de acuerdo con la Universidad Nacional Abierta y a Distancia (UNAD), se define como el proceso de apropiación crítico de la experiencia vital, intelectual y cultural, a partir del reconocimiento de la realidad personal y social, mediante la profundización teórica de conceptos, aplicándolos a la vida cotidiana permitiéndole un desarrollo de procesos académicos y la promoción del desarrollo humano [15], lo que le permite al estudiante tener una formación basada en sus propias inquietudes $\mathrm{y}$ experiencias. Además de un aprendizaje autónomo, un OVA desarrolla el aprendizaje inclusivo, el cual permite transformar los sistemas educativos con el fin de mejorar la enseñanza en todos los niveles y ambientes para responder a los estudiantes [16].

Con las ventajas que presenta el uso de un OVA en la enseñanza, y al aplicar la metodología de la indagación para el proceso de enseñanza-aprendizaje del diseño de intercambiadores de calor tipo coraza-tubos, se une en una sola metodología tres conceptos importantes. Entonces, partiendo de la necesidad del estudiante por aprender a diseñar estas máquinas térmicas, se elabora una nueva metodología para la enseñanza-aprendizaje del diseño de intercambiadores de calor tipo coraza-tubos, basado en la metodología de la indagación y usando como herramienta un OVA, cuyo desarrollo se muestra en esta investigación.

\section{Métodos}

En esta sección del documento, se dio explicación a la forma en la que se abordó el problema y se llegó al procedimiento de enseñanza- aprendizaje apropiado, que permitió integrar la metodología de indagación, el diseño térmico y el uso de un OVA. Primero, se definieron claramente los roles de los individuos involucrados en el proceso, y las herramientas en las que se apoyó su implementación; segundo, se estableció la metodología que dio solución al problema; por último, se crearon los medios por los cuales la metodología se llevó a cabo. Los individuos involucrados en el proceso fueron el estudiante y el docente, entendiendo que, aunque exista un grupo de estudiantes, el desarrollo cognitivo es individual debido a que cada uno es responsable de su aprendizaje.

La metodología planteó que el estudiante debía tener un rol activo dentro de su propio aprendizaje, dado que es quien formula las preguntas, las cuales amplían sus nociones sobre el tema y motivan su desempeño dentro del proceso. El nuevo proceso, propuesto por los autores, no planteó preguntas dentro de su desarrollo, pero sí situaciones que llevaron a resolverlas, haciendo que el alumno fuera su propio guía dentro del proceso, siempre apoyado del profesor. Simultáneamente, se definió el 
rol del docente como activo, puesto que, brinda apoyo constante al proceso de enseñanza-aprendizaje del alumno y al desarrollo de las situaciones a las que van a dar solución. Para esto, se incentivó al docente a estar documentado sobre el diseño de intercambiadores de coraza-tubos para garantizar la correcta respuesta a cualquier pregunta que le sea formulada. Así mismo, la metodología permitió que el docente planteara al grupo de estudiantes, las preguntas que considerase pertinentes, dándole la libertad de encaminar su clase. Al responder las preguntas individuales, el profesor puede apoyar más fácilmente el desarrollo de cada uno de los estudiantes, brindándole la posibilidad de conocer el proceso de enseñanzaaprendizaje y tomar decisiones al respecto. La nueva metodología fue implementada en una guía o protocolo, una para el estudiante y otra para el profesor, estas dos guías, se basaron en los ítems principales del programa, desarrollado en Excel, llamado Diseño de Intercambiadores de calor tipo Coraza-Tubos, de acrónimo DICT.

La nueva metodología propuesta, se basó en la solución de tres problemas desarrollados en dos modalidades. La primera modalidad, se realizó con base en la solución de dos de los tres problemas, uno de cálculo de calor total y otro de cálculo de área de trasferencia, en donde se abordaron primero de manera analítica, luego se resolvieron los mismos problemas en el programa, y por último se compararon los resultados. Para la segunda modalidad, se resolvió el tercer problema que correspondió al diseño específico de intercambiadores de calor, que se encaminó al cálculo de coeficiente de trasferencia de los dos fluidos junto con otros valores importantes dentro del diseño. Aunque el problema fue resuelto con el programa, para esta segunda modalidad también fue necesario hacer algunos cálculos previos de variables, ya que fueron solicitados por el programa, pero no fueron proporcionados directamente por el problema.

Es importante aclarar que ambas modalidades fueron elaboradas paso a paso, con el fin de explicar mejor al estudiante, sobre la manera en la que se resolvieron los problemas, para que así él pueda repetir los mismos pasos al desarrollar problemas de su propio interés. Los cuatro primeros pasos en ambas modalidades fueron los mismos, a parte de estos pasos en común, cada modalidad desarrolla sus propios procedimientos de acuerdo con la forma en la que se resolvieron los tres problemas.

En la Fig. 1, se muestra un mapa conceptual que ilustra el paso a paso que se siguieron en cada modalidad. En éste se evidencian los cuatros pasos en común de ambas modalidades, luego se ramifican mostrando los pasos que se siguieron en cada modalidad. Como se ve, ambas concluyen en un paso final común.

Como ya se estableció en el presente documento, la indagación es la manera como los estudiantes aprenden al formular y dar respuesta a las interrogantes que surjan en el aula de manera individual o grupal, de acuerdo con sus intereses, habilidades y ritmos de trabajo. En concordancia con la nueva metodología propuesta, cada paso da pie a una serie de preguntas que son las que inician el proceso indagatorio del estudiante, entonces en cada paso el alumno formula ciertas preguntas que al responderlas puede continuar con el siguiente paso de la solución del problema y así avanzar en el proceso de enseñanza-aprendizaje.
Teniendo claro los pasos de cada modalidad, se muestra cómo la metodología propuesta integró en cada uno de éstos, las tres bases de la investigación, para recordar, la metodología de indagación, el diseño térmico de intercambiadores de calor tipo coraza-tubos y un OVA. De tal forma que garantiza el proceso de enseñanza-aprendizaje que debe tener el estudiante al desarrollar los problemas de diseño de intercambiadores de calor. A continuación, se muestra una serie de tablas que desarrollan cada uno de los pasos de las dos modalidades, de tal forma que el lector tenga claro el propósito de cada uno éstos dentro de la nueva metodología propuesta por los autores. En la Tabla 1, se observa la forma en la que se realizaron los primeros pasos, comunes, de la metodología; en la Tabla 2, los pasos que desarrolló la primera modalidad, los cuales fueron expuestos en la Fig.1, y en la Tabla 3, se muestran los pasos restantes de la segunda modalidad, mencionados anteriormente.

En las tablas ya descritas, en las columnas de indagación se sugieren el tipo de preguntas que el estudiante puede proponer en cada paso, y un ejemplo de éstas. Téngase en cuenta que, como el proceso de indagación depende del individuo o del grupo, el tipo de preguntas planteado en el presente documento son una sugerencia por parte de los autores, sin embargo, éstas pueden cambiar a la hora de aplicar la metodología. También debe tenerse en cuenta que la metodología requiere de la presencia del docente las primeras veces que se aplique o se use en el aula, para así apoyar el proceso de enseñanza-aprendizaje del estudiante.

Una vez aclarada la metodología que fue diseñada, se hace necesario explicar de manera general la forma en la que se presentaron las dos guías, para el estudiante y docente, en las cuales se desarrollaron los tres problemas mencionados anteriormente. La guía del docente cuenta con la explicación de lo que se espera que haga el alumno en cada ítem, junto con algunas sugerencias que pueden ayudar en dicho desarrollo. Para la guía del estudiante, se presentó cada uno de los problemas, pero éstos acompañados únicamente de los ítems de desarrollo sin la explicación correspondiente, la cual se encuentra en la guía del docente.

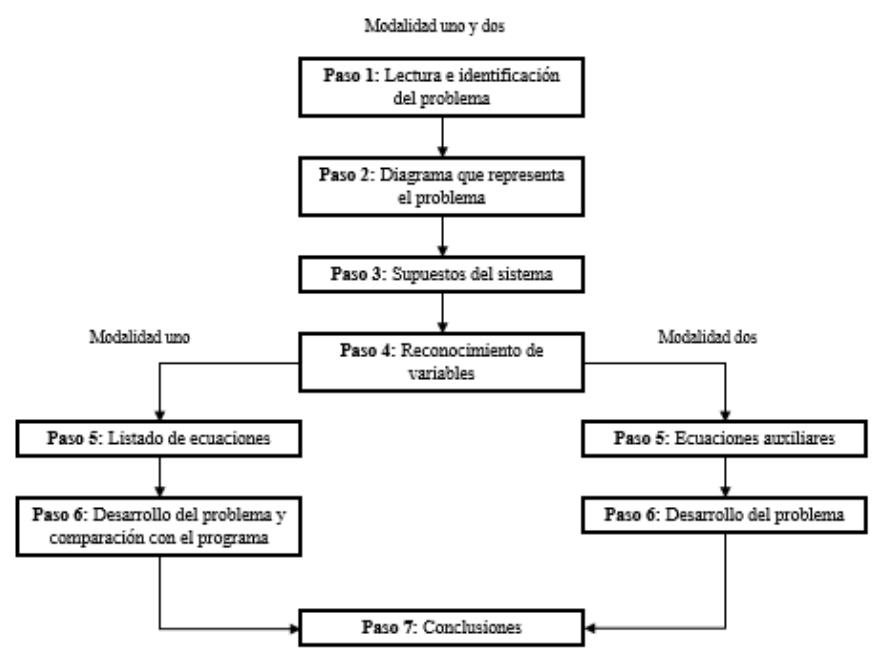

Figura 1. Desarrollo, paso a paso, de la metodología propuesta por los autores. Fuente: Los autores 
Tabla 1.

Metodología propuesta para el proceso de enseñanza-aprendizaje del diseño de intercambiadores de calor tipo coraza-tubos.

\begin{tabular}{|c|c|c|c|c|}
\hline Paso & $\begin{array}{l}\text { Descripción del proceso dentro del } \\
\text { diseño térmico }\end{array}$ & \multicolumn{2}{|r|}{ Indagación } & $\begin{array}{l}\text { Uso del programa } \\
\text { DICT }\end{array}$ \\
\hline Diagrama & $\begin{array}{l}\text { Al realizar el diagrama del } \\
\text { intercambiador se tiene en cuenta la } \\
\text { estructura general del mismo. Se } \\
\text { reconoce el flujo de ambos fluidos, } \\
\text { características de entrada y salida. }\end{array}$ & $\begin{array}{l}\text { - Estructurales } \\
\text { - Configuración } \\
\text { - Diseño } \\
\text { Intercambiadores }\end{array}$ & $\begin{array}{l}\text { - ¿Cómo se distribuyen los pasos de } \\
\text { los tubos en la coraza? } \\
\text { - ¿Qué fluido va en la coraza y cuál en } \\
\text { los tubos? ¿Cómo se determina? }\end{array}$ & No es necesario su uso. \\
\hline $\begin{array}{l}\text { Suposiciones } \\
\text { sobre } \\
\text { problema }\end{array}$ & $\begin{array}{l}\text { Se hacen suposiciones iniciales sobre el } \\
\text { sistema termodinámico antes de } \\
\text { desarrollar el problema, este ayuda a } \\
\text { delimitarlo y así poderlo resolver de una } \\
\text { forma más eficiente y rápida. }\end{array}$ & $\begin{array}{l}\text { - } \text { Conceptual } \\
\text { - Termodinámico } \\
\text { - Estructural } \\
\text { - Transferencia de } \\
\text { calor* } \\
\end{array}$ & $\begin{array}{l}\text { - ¿Qué tipo de suposiciones se } \\
\text { realizan? } \\
\text { - ¿Qué implica que el sistema esté en } \\
\text { estado estable o transitorio? * }\end{array}$ & No es necesario \\
\hline
\end{tabular}

Fuente: Los autores

Nota: * Dependiendo de las suposiciones hechas para cada problema, habrá cierto tipo de preguntas.

Tabla 2.

Metodología propuesta para el proceso de enseñanza-aprendizaje del diseño de intercambiadores de calor tipo coraza-tubos, primera modalidad.

\begin{tabular}{|c|c|c|c|c|}
\hline \multirow{2}{*}{ Paso } & \multirow{2}{*}{$\begin{array}{c}\text { Descripción del proceso dentro del } \\
\text { diseño térmico }\end{array}$} & \multicolumn{2}{|c|}{ Indagación } & \multirow{2}{*}{$\begin{array}{c}\text { Uso del programa } \\
\text { DICT }\end{array}$} \\
\hline & & Tipo de preguntas & Ejemplos & \\
\hline $\begin{array}{l}\text { Desarrollo del } \\
\text { problema }\end{array}$ & $\begin{array}{l}\text { De acuerdo con todos los elementos } \\
\text { desarrollados en los pasos } \\
\text { anteriores, el estudiante procederá a } \\
\text { desarrollar el problema y hallar la } \\
\text { solución del mismo. }\end{array}$ & $\begin{array}{l}\text { Puede formularse de todos } \\
\text { los tipos ya mencionados. }\end{array}$ & $\begin{array}{l}\text { - ¿De qué manera se deben iniciar } \\
\text { los cálculos? } \\
\text { - ¿Cómo aplican las suposiciones } \\
\text { dentro de los cálculos? } \\
\text { - ¿¿or qué al comparar mis } \\
\text { resultados hallados con el } \\
\text { método analítico, difieren con } \\
\text { los del programa? * }\end{array}$ & $\begin{array}{l}\text { En este punto es donde } \\
\text { más se utiliza, ya que a } \\
\text { medida que va } \\
\text { resolviendo cada } \\
\text { ecuación, va comparando } \\
\text { sus resultados con los } \\
\text { arrojados por el } \\
\text { programa. Además, le } \\
\text { brinda una metodología u } \\
\text { orden en el desarrollo de } \\
\text { las ecuaciones. }\end{array}$ \\
\hline
\end{tabular}

Fuente: Los autores

Nota: *Estas preguntas no son tan específicas como las que puede realizar el estudiante, pero van a depender del problema y sus componentes.

Tabla 3.

Metodología propuesta para el proceso de enseñanza-aprendizaje del diseño de intercambiadores de calor tipo coraza-tubos, segunda modalidad.

\begin{tabular}{|c|c|c|c|c|}
\hline \multirow{2}{*}{ Paso } & \multirow{2}{*}{$\begin{array}{l}\text { Descripción del proceso } \\
\text { dentro del diseño térmico }\end{array}$} & \multicolumn{2}{|c|}{ Indagación } & \multirow{2}{*}{$\begin{array}{c}\text { Uso del programa } \\
\text { DICT }\end{array}$} \\
\hline & & $\begin{array}{l}\text { Tipo de preguntas } \\
\end{array}$ & Ejemplo & \\
\hline
\end{tabular}




\begin{tabular}{|c|c|c|c|c|}
\hline $\begin{array}{l}\text { Desarrollo del } \\
\text { problema }\end{array}$ & $\begin{array}{l}\text { Junto con todos los componentes ya } \\
\text { desarrollados, el programa va a arrojar los } \\
\text { resultados requeridos por el problema. }\end{array}$ & $\begin{array}{l}\text { - Diseño de } \\
\text { intercambiadores. }\end{array}$ & $\begin{array}{l}\text { - ¿Son coherentes los valores } \\
\text { obtenidos? } \\
\text { - ¿Todos los valores que arroja e } \\
\text { programa son útiles para } \\
\text { resolver el problema? }\end{array}$ & $\begin{array}{l}\text { El programa es el } \\
\text { que arroja los } \\
\text { valores que más } \\
\text { adelante el } \\
\text { estudiante va a } \\
\text { interpretar. } \\
\end{array}$ \\
\hline \multirow{2}{*}{ Paso } & \multirow{2}{*}{ Descripción paso dentro del diseño térmico } & \multicolumn{2}{|c|}{ Indagación } & Uso del programa \\
\hline & & $\begin{array}{l}\text { Tipo de preguntas } \\
\end{array}$ & Ejemplo & DICT \\
\hline Conclusiones & $\begin{array}{l}\text { Es el punto más importante del proceso. } \\
\text { Determina qué debe hacer con los valores que } \\
\text { obtuvo y qué puede decir al respecto. Va más } \\
\text { allá de los resultados que obtuvo }\end{array}$ & $\begin{array}{l}\text { - Puede realizar cualquier } \\
\text { tipo, este es un ejercicio } \\
\text { que exige a la persona } \\
\text { sintetizar lo antes } \\
\text { realizado }\end{array}$ & $\begin{array}{l}\text { - ¿Puede construirse este diseño? } \\
\text { • ¿Qué costo tendría su } \\
\text { fabricación? }\end{array}$ & No aplica \\
\hline
\end{tabular}

Fuente: Los autores

\section{Resultados}

Del presente estudio, se obtuvieron tres diferentes productos, a saber, una guía para el docente, una guía para el estudiante y un programa de Diseño de Intercambiadores de Calor tipo Coraza-Tubos, llamado DICT. A continuación, se describen cada uno de estos productos.

\subsection{Guía para el docente y el estudiante}

Las guías son documentos en los que se resolvieron tres problemas de diseño de intercambiadores de calor tipo corazatubos, cada uno abordó diferentes características, esto para que el estudiante aprendiera a resolver diversos tipos de problemas. Además, se presentaron dos formas de desarrollo de la guía, en ambas se hizo uso del programa de diseño, sin embargo, se diferencia en su forma de uso.

Tanto la guía del docente como la del estudiante resolvieron los mismos problemas, se diferencian en que la primera explico paso a paso el desarrollo del problema mostrado en forma de recuadros, de tal forma que el profesor sepa que debe esperar o cómo debe guiar al estudiante durante el proceso de solución del problema. Por ejemplo, la primera situación que se planteó, en el primer paso se solicitó al estudiante leer el problema e identificar de qué se trata y sus características principales. En la Fig. 2, se observa un mapa conceptual, esto para el mejor entendimiento del lector, en donde se describe la forma en la que se presentó el primer paso de la primera solución del problema, es decir la lectura del mismo.

Como se ilustra en el mapa conceptual, en el primer paso de la guía del estudiante, se le solicitó que leyera el problema con detenimiento, para luego mostrarle lo que debió concluir rápidamente sobre la lectura que acaba de hacer. En cuanto a la guía del docente, no sólo se le mostró lo que se espera que el estudiante responda, sino que se le hicieron sugerencias sobre cómo apoyarlo en este paso, en caso que el alumno encuentre dificultades.

Como se ha venido mencionando, las guías se elaboraron con base en una serie de pasos que permitieron la solución de los ejemplos planteados. Dichos procedimientos fueron explicados detalladamente en las tablas de métodos en donde se hace la descripción del paso dentro del diseño térmico. Entonces, en la guía del estudiante como se ejemplificó en el mapa conceptual, se mostró cómo se espera que él desarrolle cada paso, en el caso de la guía del docente no sólo cuenta con lo explicado en la guía del estudiante, sino que le muestra al profesor cómo puede apoyar el proceso del alumno en cada uno de los pasos.

En la primera opción de desarrollo se propuso que el estudiante resolviera el problema de manera analítica y al final comparara sus resultados con los arrojados por el programa para así concluir con base en ella. En la segunda opción de desarrollo, el estudiante resuelve el problema directamente con el programa para así poder concluir respecto al diseño de la máquina térmica y establecer su funcionalidad. A partir de las dos guías elaboradas por los autores, el estudiante puede enfrentarse a cualquier problema de diseño de intercambiadores, bien sea de manera autónoma o con el acompañamiento de un profesor y poder resolverlo de manera sistemática y precisa. A medida que repita el proceso con más problemas, adquiere práctica y experiencia en su solución.

\subsection{Programa para el Diseño de Intercambiadores de Calor tipo Coraza-Tubos DICT}

El programa desarrollado se dividió en tres secciones, una de calor total, otra de cálculo de área de trasferencia y finalmente una sobre las características del diseño, que se divide a su vez en diseño de tubos y de coraza. A lo largo de todo el programa el usuario fue guiado de manera ordenada de tal forma que las variables fueron solicitadas en una secuencia determinada de diseño, que fueron consecuentes con el desarrollo de los problemas en las guías. A continuación, se muestra de manera resumida la forma en la que el programa solicita las variables y los datos obtenidos en cada una de las secciones.

En la Fig. 3, se puede observar el cálculo de calor total del intercambiador de calor, las variables requeridas fueron las temperaturas de los fluidos, el tipo de fluido que se van a usar para el intercambio de calor, para así determinar el coeficiente total de trasferencia, y el área superficial de transferencia entre otros. Con estos valores, el programa calcula la temperatura media logarítmica, el factor de corrección de la máquina y el calor total transferido. 


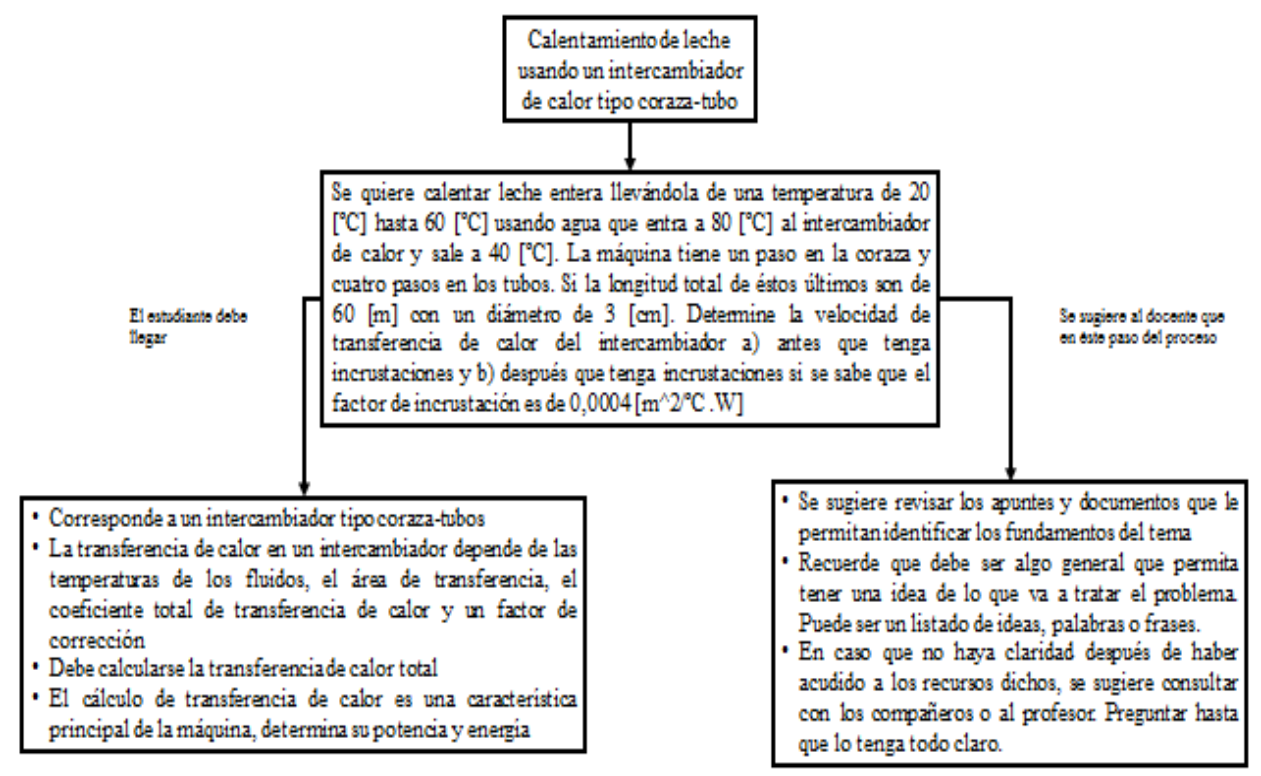

Figura 2. Mapa conceptual sobre el desarrollo de la guía, primera forma de resolver, paso uno.

Fuente: Los autores

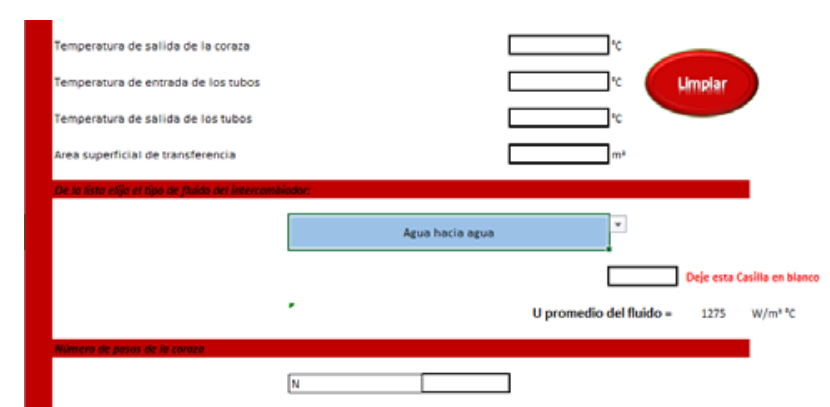

Figura 3. Fragmento del programa DICT, para el cálculo de calor total de transferencia.

Fuente: Los autores

En la Fig. 4, se muestra un fragmento del cálculo del área superficial de trasferencia de calor en donde se solicitó al usuario que eligiera el tipo de fluidos que iba a utilizar en la máquina, los flujos másicos y las temperaturas de los mismos, además de los calores específico. Ya que para este cálculo es necesario el calor total de trasferencia el programa solicita dicho valor, en caso que el usuario no lo tenga este le da la opción de calcularlo como se observa en la figura. Dentro de los valores que se obtiene, al igual que el cálculo del calor total de trasferencia, está la temperatura media logarítmica y el factor de corrección de la máquina, además del área superficial de transferencia.

En las Figs. 4 y 5, se muestran los dos cálculos relevantes de las características del diseño, el diseño de tubos y el diseño de coraza. Para el diseño de tuberías el programa requiere como variables el área superficial de trasferencia, el diámetro de la tubería y la longitud, entre otros. Derivado de este valor se obtiene el número de tubos, número de Reynolds y velocidad de flujo además de otros valores relevantes. Para el diseño de coraza, el programa requiere de los diámetros exterior e interior de la coraza, el flujo másico del fluido que pasa por la coraza, con lo cual calcula el área trasversal de esta parte del intercambiador de calor. También solicita la viscosidad del fluido, con lo que calcula el número de Reynolds y el factor de corrección del intercambiador junto con otros valores relevantes en el diseño final.

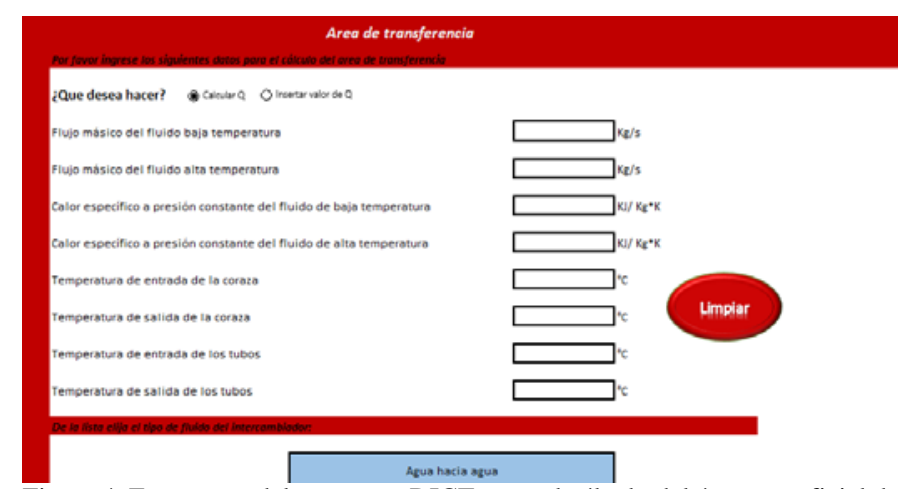

Figura 4. Fragmento del programa DICT, para el cálculo del área superficial de transferencia.

Fuente: Elaborado por los autores

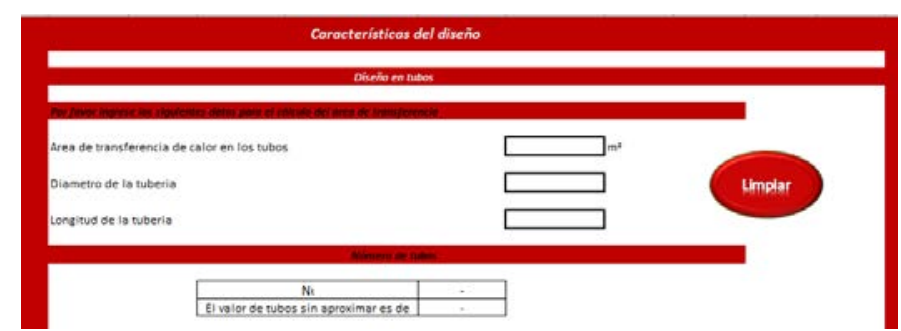

Figura 5. Fragmento del programa DICT, para las características del diseño, específicamente el diseño de tubos.

Fuente: Los autores. 


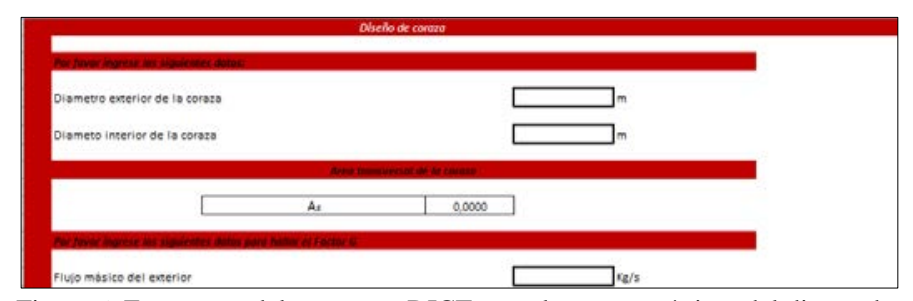

Figura 6. Fragmento del programa DICT, para las características del diseño de la coraza.

Fuente: Los autores

A lo largo del programa se encuentra el botón "limpiar" que permite vaciar los datos suministrados y hacer nuevamente los cálculos. A demás, tiene la posibilidad de diseñar en sistema internacional y en sistema inglés.

El conjunto de las guías y el programa DICT elaborados de la manera en que se mostraron, permiten reunir de una forma concreta la metodología de la indagación, el diseño térmico de un intercambiador de calor tipo coraza-tubos y un OVA.

\subsection{Implementación de una guía rápida usando la metodología propuesta para el proceso de enseñanza- aprendizaje del diseño de intercambiadores de calor tipo coraza-tubo}

A continuación, se muestra la aplicación de la metodología propuesta para el proceso de enseñanza-aprendizaje del diseño de intercambiadores de calor tipo coraza-tubo. Entonces, en primera medida se describe la población a la cual fue aplicada la guía, seguidamente se describe la manera en la que se implementa la guía y finalmente se conoce los resultados y percepciones de los estudiantes respecto a la guía.

Es importante tener en cuenta, antes de hacer las correspondientes descripciones, que la guía que es implementada a este grupo de estudiantes es una guía rápida basada en la guía original elaborada por los autores, esto se debe a que, al no tener registros bibliográficos sobre la implementación de una metodología similar a la propuesta, la prueba que se realizar es un acercamiento a lo que resultar la implementación de la metodología en contextos educativos reales por primera vez. Esta guía rápida es implementada bajo la supervisión y asesoramiento de uno de los autores.

Ya que la metodología es elaborada especialmente para estudiantes de pregrado en ingeniería mecánica que hayan cursado trasferencia de calor, o que lo estén cursando pero que se encuentren estudiando el tema de diseño de intercambiadores de calor o que ya hayan concluido el tema, se elige al grupo de estudiantes que cursan laboratorio de transferencia de calor en la Universidad del Valle en el segundo semestre del año 2017, quienes ya han aprobado la materia teórica y se encuentran cursando el laboratorio. Al ser un grupo de once personas, la población es lo suficientemente pequeña como para realizar un censo, por lo cual se convocó un día al grupo para realizar la primera prueba de la metodología.

En el día concretado por los estudiantes y el autor, sólo se presenta uno de los once estudiantes, por lo cual se elabora la prueba con esa persona. En primer lugar, se le explica que se va a resolver un problema de diseño de intercambiadores de calor tipo coraza-tubo teniendo en cuenta una serie de pasos, que son los que corresponden a la nueva metodología, para darle solución.

Al seguir cada uno de los pasos, el estudiante plantea preguntas relacionadas con el desarrollo del problema, bien sea conceptuales o prácticas, a las cuales el autor responde y aclara, esto para continuar con el desarrollo del problema. Así mismo, en cada paso el autor le formula preguntas al estudiante, de tal forma que el ratifica si entiende o no con claridad la respuesta la pregunta que le formularon. Una vez finalizado el desarrollo analítico del problema, se hace uso del programa DICT para comprobar si el resultado al que llegó el estudiante es correcto o no. Se concluye que los resultados son los mismos y que se llegó a la respuesta deseada. Luego de desarrollar este problema, se resuelve otro usando exclusivamente el programa de diseño DICT. Todo el proceso descrito duró una hora, ya que el estudiante tenía muchas dudas respecto al tema y se requirió de una explicación extensa del tema.

Una vez resueltos los dos problemas, se le presenta una encuesta sobre su percepción sobre la implementación de los pasos en la solución del problema y del uso del programa de diseño. Se le pide que califique unas afirmaciones de uno a cinco, siendo uno "en total desacuerdo" y cinco "totalmente de acuerdo". A continuación, se muestra en la Tabla 4, las afirmaciones y la puntuación que es estudiante da sobre ellas.

Tabla 4

Encuesta hecha a estudiante sobre la nueva metodología a la hora de resolver un problema.

\begin{tabular}{|c|c|}
\hline $\begin{array}{l}\text { Afirmaciones respecto a los pasos } \\
\text { de la metodología y la } \\
\text { implementación del programa }\end{array}$ & Calificación del estudiante \\
\hline $\begin{array}{l}\text { La lectura detenida del problema es } \\
\text { pertinente para su desarrollo y } \\
\text { entendimiento. }\end{array}$ & 5 \\
\hline $\begin{array}{l}\text { La elaboración del diagrama que } \\
\text { representa el problema es } \\
\text { importante para su mejor } \\
\text { entendimiento. }\end{array}$ & 5 \\
\hline $\begin{array}{l}\text { Los supuestos del sistema permiten } \\
\text { conocer las condiciones del mismo y } \\
\text { así poder encaminar el problema de } \\
\text { una manera correcta. }\end{array}$ & 5 \\
\hline $\begin{array}{l}\text { El reconocimiento de las variables } \\
\text { conocidas y desconocidas permite } \\
\text { identificar qué se espera identificar } \\
\text { con el desarrollo del problema, y } \\
\text { qué información me proporcionan } \\
\text { para cumplir el objetivo. }\end{array}$ & 5 \\
\hline $\begin{array}{l}\text { Elaborar un listado de ecuaciones } \\
\text { me permite reconocer la manera en } \\
\text { la que las variables se relacionan. }\end{array}$ & 5 \\
\hline $\begin{array}{l}\text { Con los pasos que se llevaron a cabo } \\
\text { previamente pude resolver } \\
\text { fácilmente el problema. }\end{array}$ & 4 \\
\hline $\begin{array}{l}\text { En cada paso surgieron preguntas a } \\
\text { nivel individual o grupal, que fueron } \\
\text { resultas a lo largo del desarrollo. }\end{array}$ & 4 \\
\hline $\begin{array}{l}\text { Aclaré dudas conceptuales que tenía } \\
\text { sobre el diseño de intercambiadores } \\
\text { de calor tipo coraza-tubo. }\end{array}$ & 5 \\
\hline $\begin{array}{l}\text { El uso del programa DICT } \\
\text { complementa el desarrollo del } \\
\text { problema y permite aclarar dudas }\end{array}$ & 5 \\
\hline
\end{tabular}




\section{Discusión}

Al obtener como resultado dos guías o protocolos y un programa de diseño de intercambiadores, se muestra la forma en la que las herramientas pedagógicas e ingenieriles pueden unirse en beneficio de la formación de ingenieros mecánicos. La comprensión profunda de la manera en la que se abordan los problemas propuestos en el aula, y no su resolución mecánica o rutinaria, que permite esta nueva metodología, son los que ayudan a desarrollar en el ingeniero el pensamiento lógico y crítico necesario para resolver las dificultades que se le presenten en su vida laboral.

Con base en este desarrollo de pensamiento, las guías son una herramienta útil tanto para el estudiante como para el docente, ya que les proporciona un acompañamiento continuo en el proceso de enseñanza-aprendizaje, de tal forma que el estudiante sabe hacia dónde se dirige la solución del problema y el docente comprende la forma en la que debe apoyar al estudiante en el proceso. En relación al programa de diseño, es una herramienta visual para los usuarios ya que les permite profundizar en el tema del diseño de intercambiadores de calor tipo coraza-tubos. Aunque en el aula no se profundice en el diseño térmico de este tipo específico de dispositivos, con el uso del programa el estudiante puede aproximarse de manera general a los factores y cálculos que requiere este tipo de diseño.

Este primer artículo, pretende dar a conocer a la comunidad científica, los fundamentos del nuevo método de enseñanzaaprendizaje, el cual se fundamenta en la metodología de la indagación, el diseño térmico de intercambiadores y la aplicación de un objeto virtual de aprendizaje. Actualmente, el nuevo método está siendo implementado y monitoreado en un grupo de estudiantes de transferencia de calor, mediante el uso de las dos guías de enseñanza, una para el estudiante y otra para el profesor, y un programa de diseño de intercambiadores de calor. Esto con el objetivo de realizar un estudio robusto donde se obtenga cuantitativamente los beneficios del nuevo método para la enseñanza de intercambiadores de calor.

Por los motivos anteriores, en esta publicación solo se describen cualitativamente la implantación del método, en los estudiantes mencionados. Según las respuestas de una encuesta aplicada al grupo de estudiantes de transferencia de calor, se evidencia que el uso de la metodología es una herramienta útil en la solución de los problemas planteados en la guía del estudiante.

La metodología de la indagación, el diseño térmico de intercambiadores de calor tipo coraza-tubos y el uso de un OVA, no son términos nuevos dentro de la pedagogía y la ingeniería, sin embargo, no se había propuesto una manera en la que los tres conceptos se unieran a favor de un mejor proceso de enseñanza-aprendizaje en la formación de nuevos ingenieros mecánicos. Es por esto que, la estructura de la nueva metodología propuesta plantea una base en el desarrollo del pensamiento ingenieril, el cual permite formar profesionales competentes en su área de trabajo.

\section{Referencias}

[1] CODELCO, Aprender y enseñar ciencias a través de la indagación, en: 2do Encuentro Educación y Minería, 2008.
[2] Harlen, W., Evaluación y educación en ciencias basada en la indagación: Aspectos de la política y la práctica, 2013.

[3] The Global Network of Science Academies, Report of the IAP Science Education Program Taking Inquiry-Based Science Education into Secondary Education A global conference, 2010.

[4] National Research Council (NRC), National Science Education Standards. Washington DC, 1996.

[5] National Science Foundation (NSF), The Challenge and Promise of K-8 Science Education Reform. Arlington, 1997.

[6] Cengel, Y.A. and Ghajar, A., Heat and mass transfer. Fundamentals and Applications. 2014.

[7] Sukhatme, S.P., A textbook on heat transfer, 4th ed. 2005.

[8] Serth, R.W., Process heat transfer. Principles and applications, 2 ed.2007.

[9] Aguilar, R. and Rafael, F., Metodología para el diseño de un intercambiador de calor de tubo de coraza, Rev. Inst. Politécnico Nac., 2, pp. 56-75, 2010.

[10] Fonseca, L. and Riveros, L., Diseño térmico y mecánico de intercambiadores de calor de casco y tubo, Tesis de grado, Universidad Industrial de Santander, Bucaramanga, Colombia, 2009.

[11] Parra-Castrillón, E., Propuesta de metodología de desarrollo de software para objetos virtuales de aprendizaje -MESOVA, Rev. Virtual Univ. Católica del Norte, 2011.

[12] Ortega-Barba, C.F., Tecnologías de la información y la comunicación para la innovación educativa. En: Ruiz-Velasco E., (coordinador). México, CONAC y T/UNAM -Posgrado Pedagogía/Díaz de Santos Colección Estudios, 2012. Perfiles Educativos [en linea] 2014, XXXVI. Disponible en: http://www.redalyc.org/articulo.oa?id=13230751014.

[13] Jiménez-García, F.N., Márquez-Narváez, C., Agudelo-Calle, J.D.J. and Beleño, L., Una experiencia didáctica en el diseño e implementación de objetos de aprendizaje para la enseñanza de la física, Revista Educación en Ingeniería, 11(22), pp. 13--20, 2016.

[14] Jara, C., Real-time collaboration of virtual laboratories through the Internet, Comput. Educ., 52(1), pp. 126-140, 2009.

[15] Universidad Nacional Abierta y a Distancia UNAD, Proyecto Académico Pedagógico Solidario. 2011.

[16] Rojas, F., Ingeniero de inclusión social, madres cabeza de hogar, LACCEI. Guayaquil - Ecuador, 2014.

S.A. Carrión-Avellaneda, recibió el título de Ing. Mecánico en 2006, de la Universidad Nacional de Colombia, en la sede Bogotá. Actualmente, es estudiante de Maestría en Ingeniería Mecánica en la Universidad Nacional de Colombia, en la sede Bogotá. Ha sido profesor en el área de ciencias térmicas, especialmente dirigiendo las asignaturas de termodinámica y transferencia de calor. Sus áreas de actuación son la educación en ingeniería, dinámica de fluidos computacional y el modelado de combustibles.

ORCID: 0000-0001-7748-7302

L.A. Ardila-Castro, recibió el título de Ing. Mecánica en septiembre de 2016 de la Escuela Colombiana de Ingeniería Julio Garavito, con énfasis en Gestión Eficiente y Sostenible de la Energía. Actualmente es estudiante de Maestría en Ingeniería con énfasis en Ingeniería Mecánica en la Universidad del Valle. Ha realizado un trabajo investigativo realizado sobre el diseño e instalación de laboratorios de vapor. Tiene experiencia docente en física y estadística. Se encuentra actualmente vinculada al grupo de investigación de Dinámica de Fluidos en la línea de investigación de Energías Renovables no Convencionales de la Universidad del Valle.

ORCID: 0000-0001-9708-3751

C.A. Ramírez-Mendoza, es estudiante de décimo semestre de Ing. Industrial en la Escuela Colombiana de Ingeniería Julio Garavito. Actualmente es miembro y líder del semillero de investigación en BPM (Business Process Management) del cual obtiene como resultado en el año 2017 la publicación y ponencia del artículo titulado "Estudio sobre la Cultura Nacional y Organizacional en Colombia para Favorecer la Formación de Perfiles más Afines con las Necesidades Organizacionales" en el Encuentro Internacional de Educación en Ingeniería ACOFI 2017.

ORCID: 0000-0002-1323-9796 